eat raw larvae or pupae of wasps.

Kantha suggested insectivory could both provide a source of animal protein and remove agricultural pests. But another possibility is that insects are a source of salt and minerals. Nanshin is surrounded by mountains $2,000-3,000$ metres high, and has heavy annual rainfall. These conditions must have made it difficult to bring in salt from coastal districts when traffic networks were still poorly developed.

A teacher at a high school in Nanshin tells me that until about 100 years ago, when the production of salt was reduced in coastal districts due to humid weather conditions, the people of Nanshin frequently either ate more insects to increase their salt intake or extracted salt by boiling tatami.

Katsuya Ichinose The Institute of Low Temperature Science, Hokkaido University,

Sapporo 060, Japan

\section{Superfamily expands}

SIR - We report that two proteins of Epstein-Barr virus (EBV) are members of the immunoglobulin superfamily. The proteins were identified as immunoglobulin superfamily members by a program that can search for immunoglobulin-like domains in protein sequences. The similarity of the domains is mainly in the regions around two cysteines which form the conserved disulphide bond ${ }^{1-3}$.

The algorithm of the program is the pattern-match method of Harr et al. ${ }^{4}$. First, two fragments that consist of the 16 amino-acid residues to the right of the first disulphide bond-forming cysteines and the 16 residues to the left of the second disulphide bond-forming cysteine of 49 representative immunoglobulin-like domains were tabled (data not shown). We wrote a statistical program to calculate the frequencies of 20 residues in each position of these fragments.

Based on those data, we wrote another program that could find immunoglobinlike domains in Pascal and used it to search the PIR database (NBRF release 11.0 ) at a significance value of $10^{-16}$. Of 3,606 sequences searched, we identified 279 known members of the immunoglobulin superfamily together with six previously unrecognized members. Two of these are EBV proteins.

One is the hypothetical BARF1 protein (NBRF code QQBE48), which is encoded by an open reading frame in BamHI-A fragment of the virus genome ${ }^{5}$. The other (QQBE4L) is a probable glycoprotein. These proteins have significance values of $9.8 \times 10^{-16}$ and $8.9 \times 10^{-15}$, respectively. Local homology searches and secondary structure prediction with IDEA programs $^{16}$ confirm that residues $13-124$ of QQBE48 and residues 20-135 of QQBE4L are similar to immunoglobulin

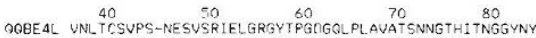

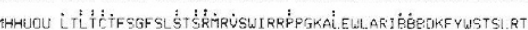
QGBE 4L SLTLËUNDOSNTSUSLII PNUT-LAHAGYYTCNUTLRNCSVASGVHCNY 110

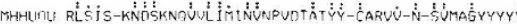

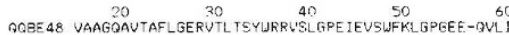

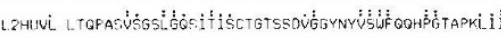 \\ GGBE48 GRMHHOUIF: 70 EWPFRGFFOHRSANTFFI. UUTAANISHOGNYL

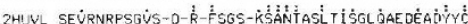

Two fragments of QQBE4L and QQBE48 aligned with two immunoglobulin variable regions (NBRF codes MHHUOU and L2HUVL). Identical residues (:) and conservative replacements (.) are highlighted. QQBE48 and QQBE4AL are related to another 18 and 11 members of the superfamily, respectively, with s.d. values above 3 . Top, homology score $=-70$, per cent match $=26.3$, s.d. $=5.865$. Bottom, homology score $=-79$, per cent match $=23.1$, s.d. $=6.285$.

variable regions and are predicted to have an immunoglobulin-fold structure (see figure). Furthermore, A. Williams (personal communication) has suggested that QQBE48 has a second domain (residues 126-221) distantly related to immunoglobulin constant domains.

The functions of the two proteins are unknown, and their immunoglobulin-like domain is unexplained. It is possible that EBV has at some stage integrated an immunoglobulin gene from its host B cell into its genome. Alternatively, one or both of the proteins may adhere to major histocompatibility complex molecules (or other molecules having an immunoglobulin-like domain) on the B-cell surface to increase the infectivity of the virus. This would resemble the way that most members of the superfamily perform their functions, such as cell recognition and adhesion, by means of associations of their immunoglobulin-like domains ${ }^{1}$.

A case of a boy who suffered from chronic active EBV infection with Kawasaki-like disease was reported recently? The patient's peripheral lymphocytes were EBV-receptor (CD21) negative, but could be infected by EBV. It will be interesting to see if QQBE48 or QQBE4L can bind to the CD4 molecule of the boy's T cells and cause the infection.

HuAichun Wang JAJIN WU Peinsien Tang

Institute of Basic Medical Sciences,

PO Box 130 (3), Beijing 100850 ,

People's Republic of China

Williams, A.F.\& Barclay, A. N. A Rev, Immun 6, 381$405(1988)$

2. Barker, W. C., Ketcham, L. K. \& Dayhoff, M. O. in Atlas of Protein Sequence and Structure, Vol. 5 (ed. Dayhoff, M.) Suppl. 3 (Natn. Biomed. Res. Found., Washington, DC 1978)

3. Matsunaga, T \& Mori, N Scand J Immun 25, 485-495 (1987)

4. Harr R Hägoström, M \& Gustafsson, P. Nucleic Acids Res. 11, 2942-2957 (1983)

5. Baer, R. et al. Nature 310, 207-211 (1984)

6. Kanehisa, M. (ed.) IDEAS-Integrated Databases and Extended Analysis System for Nucleic Acids and Protein User Manual (Natn. Cancer Institute, Frederick, Maryland, 1987)

7. Kikuta, H. et al. Nature 333, 455-457 (1988)

\section{Lead contamination}

SIR-Markovac and Goldstein (Nature 334, 71-73; 1988) report that picomolar concentrations of lead stimulate protein kinase $\mathrm{C}$. This conclusion may not be justified, as the authors do not seem to have taken into account the contamination of biochemical reagents with lead, and other metals. I have analysed a sample of $\mathrm{MgCl}_{2} \mathrm{BDH}$ Analar Grade $\left(\mathrm{MgCl}_{2}\right.$ was a component of their assay medium for PKC) by atomic absorption spectrophotometry and find $2 \times 10^{-6} \mathrm{M}$ of lead in a $1 \mathrm{M}$ solution, equivalent to $1 \times$ $10^{-8} \mathrm{M}$ in a $5 \mathrm{mM}$ solution. This is within the manufacturer's specification. Lead contamination can also be expected in other reagents used by Markovac and Goldstein, yet they report enzyme activation over a range from $10^{-16}$ to $10^{-12} \mathrm{M}$ of lead. It is hard to believe that the lead concentration was actually in this range.

T.J.B. SimONS

Department of Physiology,

King's College London,

Strand, London WC2R $2 L S, U K$

Markovac and Goldstein RePlySimon's concerns about reagent impurities are valid and, indeed, we did receive assurance from Sigma, from whom we purchased all our reagents except for 1,2diolein ( $\mathrm{P}-\mathrm{L}$ Biochemicals), that they were lead-free at the sensitivity of the manufacturer's assay system.

The buffer used for protein kinase $\mathrm{C}$ preparation contained chelating agents to remove pre-existing cations, including calcium and lead. These chelators, present in trace amounts in our reaction mixture, should bind residual lead from reagent contamination. Because we did not perform atomic absorption analysis, we cannot be certain of the actual concentration of available lead, but the addition of $10^{-10}$ $\mathrm{M}$ lead to our basal assay system reproducibly stimulated protein kinase $\mathrm{C}$ activity, suggesting this important regulatory enzyme is highly sensitive to lead.

Jasna Markovac Gary W. Goldstein

Departments of Pediatrics and Neurology,

University of Michigan Medical

School,

Ann Arbor, Michigan 48109-0570, USA

Selective membrane correction

$\mathrm{Cd}^{2+}$ should have been printed instead of $\mathrm{Cu}^{2+}$ on lines 3 and 13 of the third paragraph of the reply by Rubinstein et al. in Nature 337, 217; 1989.

\section{Scientific Correspondence}

Scientific Correspondence is intended to provide a forum in which readers may raise points of a scientific character. They need not arise out of anything published in Nature. In any case, priority will be given to letters of less than 500 words and five references. 